\title{
Objective Short Sleep Duration is Related to the Peripheral Inflammasome Dysregulation in Patients with Chronic Insomnia
}

This article was published in the following Dove Press journal: Nature and Science of Sleep

\section{Jihui wang \\ Xiaoli Wu (DD \\ Wenjing Liang \\ Minhua chen \\ Chongbang Zhao (D) \\ Xianglan Wang (D)}

Department of Psychiatry, The Third Affiliated Hospital, Sun Yat-Sen University, Guangzhou 510630, People's Republic of China
Correspondence: Xianglan Wang Department of Psychiatry, The Third Affiliated Hospital, Sun Yat-Sen University, No. 60, Tianhe Road, Tianhe District, Guangzhou 510630, Guangdong, People's Republic of China

Tel +8602085253129

Email wxiangl@mail.sysu.edu.cn
Objective: Insomnia with objective short sleep duration (IOSSD) is associated with an increased risk of cardiovascular morbidity, diabetes, neurocognitive impairment, and mortality. Inflammation is believed to be one of the main links between IOSSD and these diseases. The role of nucleotide-binding domain and leucine-rich repeat protein-3 (NLRP3) inflammasome in inducing activation of inflammatory signaling in IOSSD is not clear. In this study, we investigated the expression of NLRP3 inflammasome in patients with IOSSD to clarify this issue.

Methods: Thirty-six patients with insomnia and 20 age- and sex-matched healthy controls were sequentially recruited. Subjects were categorized into three groups: IOSSD (sleep duration $<6 \mathrm{~h}, \mathrm{n}=20$ ), insomnia with objective normal sleep duration (IONSD, sleep duration $\geq 6 \mathrm{~h}, \mathrm{n}=16)$ and healthy controls $(\mathrm{n}=20)$. Objective sleep parameters were measured by overnight polysomnography. Peripheral NLRP3 inflammasome protein levels [NLRP3, apoptosis-associated speck-like protein containing a CARD (ASC), caspase 1] and cytokines [interleukin (IL)-1 $\beta$ and IL-18] were assessed by Western blotting and ELISA, respectively. Results: IOSSD group showed significantly increased protein expressions of ASC and caspase-1 compared to IONSD and healthy controls and significantly increased IL-18 levels compared to healthy controls. On correlation analysis, total sleep time showed an inverse correlation with NLRP3, ASC, IL-18, and IL-1 $\beta$ levels. Wake after sleep onset (WASO) showed a positive correlation with NLRP3, ASC, caspase-1, and IL-1 $\beta$ levels. N3 sleep ratio showed a significant negative correlation with NLRP3, ASC, and IL-18 levels.

Conclusion: The current study demonstrated upregulation of NLRP3 inflammasome in IOSSD. Short sleep duration, decreased slow wave sleep, and sleep fragmentation may contribute to dysregulation of NLRP3 inflammasome.

Keywords: insomnia, short sleep duration, polysomnography, fragmentation, inflammation, NLRP3

\section{Introduction}

Insomnia is the one of the most common sleep disorders and affects approximately $8-10 \%$ of the general population. ${ }^{1}$ Insomnia is a heterogeneous disease that includes many subtypes; among these, insomnia with objective short sleep duration (IOSSD) is getting increasing attention. ${ }^{2}$ Objective short sleep duration is defined as a habitual nocturnal sleep time less than six hours, as measured by objective methods, such as polysomnography (PSG). ${ }^{3}$ According to a study by Suzanne et al (2018), ${ }^{4}$ IOSSD accounts for approximately $50 \%$ of patients with insomnia. IOSSD is the most 
biologically severe phenotype of insomnia. Studies have demonstrated the association of IOSSD with physiological arousal and several chronic diseases including diabetes, ${ }^{5}$ hypertension, ${ }^{6}$ cardiovascular disease, ${ }^{7}$ neurocognitive impairment, ${ }^{8}$ and depression. ${ }^{9}$ Inflammation caused by sleep disturbance is regarded as one of main underlying mechanisms of this association. ${ }^{4}$ Several animal ${ }^{10-12}$ and human $^{13-15}$ studies have documented increased levels of biomarkers of systemic inflammation after sleep deprivation; however, the relation between inflammation and sleep loss is not well characterized.

The nucleotide-binding domain and leucine-rich repeat protein-3 (NLRP3) inflammasome is an intracellular protein complex that plays an important role in innate immunity. NLRP3 is activated by bacterial, viral, other pathogenassociated molecular patterns or host-derived dangerassociated molecular patterns (including ASC), and subsequently recruits and activates the pro-inflammatory protease caspase-1. ${ }^{16}$ Activated caspase- 1 cleaves the precursors of interleukin (IL)-1 $\beta$ and IL-18 to produce corresponding mature cytokines, which triggers the subsequent inflammatory cascade. ${ }^{16}$ NLRP3 inflammasome plays a key role in the pathogenesis of several diseases, such as type 2 diabetes, ${ }^{17}$ neurodegenerative diseases, ${ }^{18}$ and atherosclerosis. ${ }^{19}$ Studies have shown that NLRP3 inflammasome contributes to sleep regulation. ${ }^{20}$ Activation of NLRP3 inflammasome has also been reported in a canine model of chronic obstructive sleep apnea $^{21}$ and in a mice model of sleep deprivation. ${ }^{22}$ To the best of our knowledge, the relationship between NLRP3 and sleep duration in patients with insomnia is not well characterized in contemporary literature. Considering the growing evidence of inflammatory activation in patients with IOSSD, the role of NLRP3 as the link between short sleep duration and inflammatory response is worth exploring. In the present study, we investigated the relationship between the expressions of NLRP3 associated proteins and objective sleep parameters in patients with IOSSD. We hypothesized that objective short sleep duration in insomnia patients was associated with peripheral NLRP3 inflammasome dysregulation.

\section{Methods}

\section{Study Design}

This was a single-center, cross-sectional, case-control study conducted at the third Affiliated Hospital of Sun Yatsen University. The sample size was determined utilizing a null-hypothesis statistical test; the number of patients included was larger than the predicted number. This study was conducted in accordance with the Declaration of Helsinki and the protocol was approved by the institutional ethics committee. Written informed consent was obtained from all participants prior to their enrolment.

\section{Participants}

Participants were recruited through advertisements posted in hospitals and referrals from doctors in outpatient clinics. Interested individuals were invited to attend a structured interview and physical examination conducted by an experienced psychiatrist. Thirty-six patients with insomnia who qualified the criteria were finally recruited. Twenty age- and sex-matched healthy individuals who sleep well were recruited as healthy controls. The healthy control group comprised of siblings of insomnia patients, or the individuals who came to the hospital for routine physical examination. A schematic illustration of the study design is presented in Figure 1.

Inclusion criteria for patients with insomnia: age range, 18-50 years; patients who qualified the diagnostic criteria for persistent insomnia as per the Diagnostic and Statistical Manual of Mental Disorders, fifth edition (DSM-V); ${ }^{23}$ Insomnia Severity Index (ISI) score $\geq 8$; voluntary provision of written informed consent for participation.

Inclusion criteria for healthy controls: age range, 18-50 years; no complaints about sleep quality or quantity; no history of psychiatric diseases or sleep-wake disorders; voluntary provision of written informed consent for participation; ISI score $<8$.

Exclusion criteria for all participants were: presence of any sleep-wake disorder other than insomnia; presence of any serious mental disorder (eg, psychotic disorder, bipolar disorder, major depression, or substance abuse); presence of unstable or serious physical diseases; presence of any inflammatory disease that may affect the results of the study; use of alcohol or medication as a sleep aid in the two-week period immediately preceding the enrolment; pregnant women; individuals engaged in night-shift work; difficulty in completing PSG or blood test.

\section{Measures}

PSG

PSG (Cadwell Laboratories, Inc, America) was used to monitor sleep for two consecutive nights in the sleep laboratory. The first night was intended to facilitate adaptation of the participants to the laboratory environment. Only the data pertaining to the second night were included in the analysis. 


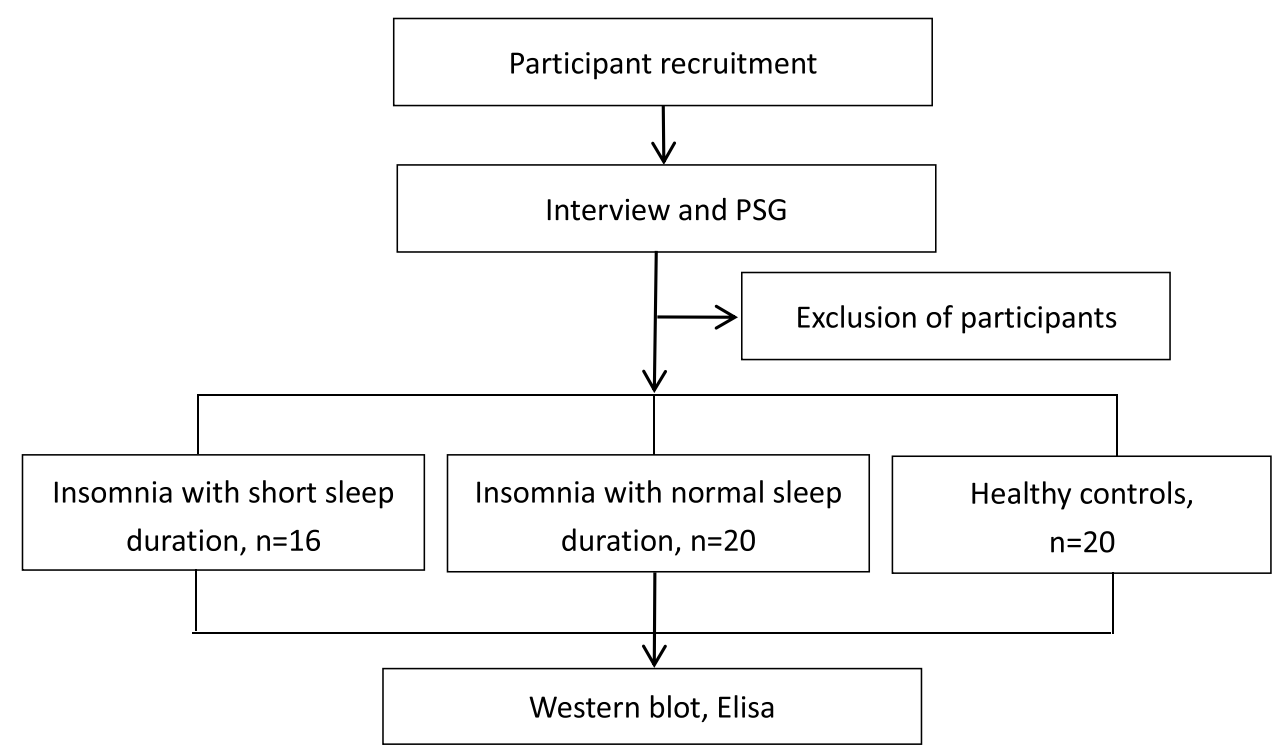

Figure I Schematic illustration of the study design. Participants were recruited through advertisements posted in hospitals and referrals from doctors in outpatient clinics. Interested individuals were invited to attend a structured interview and PSG conducted by an experienced psychiatrist. Thirty-six patients with insomnia who qualified the criteria were finally recruited. Twenty age and sex-matched healthy individuals who sleep well were recruited as healthy controls.

The participants were informed to avoid alcohol, coffee, as well as day-time sleep prior to the PSG test. PSG monitoring was started at 22:00 Hrs and lasted for at least 8 hours. An experienced psychiatrist who was blinded to the group assignment scored the PSG data according to the AASM guidelines. ${ }^{24}$ PSG-derived variables included: sleep onset latency (SOL); total sleep time (TST); sleep efficiency \% (SE \%); wake after sleep onset (WASO); percentage duration of sleep stages [ie, N1, N2, N3, rapid eye-movement (REM) sleep]; apnea hypopnea index (AHI) and arousal index (ArI). Sleep fragmentation refers to increased night awakenings and decreased sleep continuity with increased ArI and WASO, as assessed by PSG. Slow-wave sleep (SWS) is defined as sleep stages 3 and 4 that characteristically show slow delta EEG activity during PSG. PSG-TST from the second night of recording was used to categorize the insomnia patients into two sub-groups: insomnia with objective normal sleep duration (IONSD, PSG-defined TST $\geq 6 \mathrm{~h}, \mathrm{n}=16$ ) and insomnia with objective short sleep duration (IOSSD, PSG-defined TST $<6 \mathrm{~h}, \mathrm{n}=20$ ).

\section{NLRP3 Inflammasome Protein Determination Isolation of Peripheral Blood Mononuclear Cells}

Ten-milliliter fasting peripheral venous blood samples were collected in the morning after the second PSG test. The blood was centrifuged at $2000 \mathrm{r} / \mathrm{min}$ for $20 \mathrm{~min}$ at room temperature; the serum and the buffy coat were collected separately and stored at $-80^{\circ} \mathrm{C}$ until further processing. Mononuclear cells were isolated from the lymphocyte lysate using the Dynabeads $^{\circledR} \quad$ Untouched $^{\mathrm{TM}}$ Human Monocytes kit (Invitrogen; Thermo Fisher Scientific, Inc.).

\section{Western Blotting}

Mononuclear cells were washed thrice with PBS solution (Invitrogen; Thermo Fisher Scientific, Inc.) and lysed in icecold lysis buffer (Absin Bioscience Inc., Shanghai, China). Quantitative analysis of protein concentrations was performed using bicinchoninic acid kit (Shanghai Weimeng Biotechnology Co. Ltd., Shanghai, China). The extracted proteins were added to SDS-polyacrylamide gel electrophoresis $(12.5 \%$ gel) and transferred onto a nitrocellulose membrane. After blocking with 5\% skim milk powder in TBST (Tris-buffered saline with $0.1 \%$ Tween 20 ), the membranes were incubated overnight at $4{ }^{\circ} \mathrm{C}$ with the following primary antibodies: anti-NLRP3, anti-caspase 1 (1:1000, rabbit, Absin Bioscience Inc., Shanghai, China), anti-ASC, and antiGAPDH antibodies (1:1000, rabbit, Absin Bioscience Inc., Shanghai, China). After washing, blots were incubated with secondary antibodies conjugated to HRP. The signals on the membrane were detected with enhanced chemiluminescence (Cell Signaling Technology).

\section{ELISA}

Serum concentrations of IL- $1 \beta$ and IL-18 were determined using enzyme-linked immunosorbent assay (ELISA) kits (USA R\&D Systems, Inc., Minneapolis, USA) on 
a Luminex 200 system (Luminex Corporation, Texas, USA) according to the manufacturer's instructions.

\section{Statistical Methods}

SPSS 20.0 was used for statistical analysis. Data are presented as mean \pm standard deviation (SD). Sleep parameters were compared using the student's $t$-test, while NLRP3 inflammasome-associated proteins were compared using Mann-Whitney $U$-test. Correlation matrix analysis was performed to explore the relationship of PSG parameters with NLRP3 inflammasome-associated protein levels in patients with insomnia. $P$-values $<0.05$ were considered indicative of statistical significance.

\section{Results}

\section{Demographic and Clinical Characteristics of Participants}

Sixty consecutive patients with insomnia complaints were screened for recruitment. Of these, 24 patients were excluded because of the following reasons: presence of other sleep-wake disorders $(n=7)$; presence of mood disorders $(\mathrm{n}=11)$; presence of unstable medical conditions $(n=2)$; patients taking alcohol to aid in sleep $(n=2)$; (4) patients unable to complete the PSG $(n=2)$. A total of 36 insomnia patients and 20 healthy controls were finally enrolled in the study. Among the insomnia patients, 20 had normal sleep duration and 16 had short sleep duration based on the PSG test. There were no significant amonggroup differences with respect to demographic or clinical characteristics $(p>0.05)$ (Table 1).

\section{Comparisons of PSG Measures Between the Three Groups}

Compared to the IONSD and the controls, patients with IOSSD showed significantly increased SOL, WASO, ArI, and significantly decreased TST, SE, N3 and REM sleep ratio $(p<0.05)$. These findings indicated that the IOSSD group not only had short sleep duration, but also had more serious disruption of sleep continuity and slow wave sleep (SWS) (Table 2).

\section{Comparisons of Protein Expressions of NLRP3 Inflammasomes and Related \\ Cytokines}

The Western blots of NLRP3 inflammasome proteins are shown in Figure 2. There was a significant difference between the three groups with respect to the expressions of NLRP3, ASC, caspase-1, IL-1 $\beta$, and IL-18 (P < 0.05). The IOSSD group showed increased protein expressions of ASC and caspase-1 compared to IONSD and controls; the IOSSD and IONSD groups showed increased expressions of NLRP3 and IL- $1 \beta$ than controls, while the difference between IOSSD and IONSD in this respect was not statistically significant. The IOSSD group showed increased IL-18 expression compared to the controls (Figure 2). The results indicated that objective short sleep duration in insomnia patients was related to the peripheral upregulation of NLRP3 inflammasome-related proteins.

\section{Correlation Matrix Analysis of PSG Parameters and Inflammasome Related Protein Levels in Patients with Insomnia} On correlation matrix analysis, TST showed an inverse correlation with NLRP3, ASC, IL-18, and IL-1 $\beta$ levels. WASO showed a positive correlation with NLRP3, ASC, caspase-1, and IL-1 $\beta$ levels. In addition, N3 sleep ratio showed a significant inverse correlation with NLRP3, ASC, and IL-18 levels (Table 3). The results indicated

Table I Demographic and Clinical Characteristics of the Study Population Disaggregated by Groups

\begin{tabular}{|c|c|c|c|c|c|}
\hline Variables & IOSSD, $n=16$ & IONSD, $\mathrm{n}=\mathbf{2 0}$ & Controls, $n=20$ & $\mathbf{F} / \chi^{2}$ & $P$ \\
\hline Age (years), mean (SD) & $30.19 \pm 8.50$ & $30.70 \pm 8.34$ & $29.32 \pm 7.53$ & $1.208^{\mathrm{a}}$ & 0.307 \\
\hline Body mass index $\left(\mathrm{kg} / \mathrm{m}^{2}\right)$ & $21.67 \pm 2.61$ & $21.27 \pm 2.31$ & $20.48 \pm 2.49$ & $1.124^{\mathrm{a}}$ & 0.333 \\
\hline Male, n (\%) & 7 (43.8\%) & $9(45.0 \%)$ & II (55.0\%) & $0.579^{b}$ & 0.749 \\
\hline Single, $n(\%)$ & $8(50.0 \%)$ & $9(45.0 \%)$ & $10(50.0 \%)$ & $0.129^{b}$ & 0.938 \\
\hline Education $\geq 12$-year, n (\%) & $13(81.2 \%)$ & $15(75.0 \%)$ & 14 (70.0\%) & $0.600^{b}$ & $0.74 I$ \\
\hline Current smokers, n (\%) & $3(18.8 \%)$ & $3(15.0 \%)$ & $4(20.0 \%)$ & $0.183^{b}$ & 0.913 \\
\hline Insomnia duration & $6.63 \pm 4.27$ & $6.40 \pm 3.07$ & - & $0.184^{c}$ & 0.855 \\
\hline Insomnia severity index & $17.63 \pm 3.32$ & $16.55 \pm 4.88$ & - & $0.75 I^{c}$ & 0.458 \\
\hline
\end{tabular}

Notes: ${ }^{a} F$ value, ${ }^{b} \chi^{2}$ value, ${ }^{c} t$ value.

Abbreviations: IOSSD, insomnia with objective short sleep duration; IONSD, insomnia with objective normal sleep duration; SD, standard deviation. 
Table 2 Comparison of PSG Measures Between the Three Groups

\begin{tabular}{|l|l|l|l|l|l|}
\hline Variables & IOSSD, $\mathbf{n}=\mathbf{1 6}$ & IONSD, $\mathbf{n}=\mathbf{2 0}$ & Controls, $\mathbf{n}=\mathbf{2 0}$ & $\boldsymbol{F}$ \\
\hline SOL, min & $91.25 \pm 45.26^{\mathrm{AB}}$ & $64.75 \pm 27.08^{\mathrm{C}}$ & $28.75 \pm 20.70$ & $\mathbf{P}$ \\
TST, min & $251.25 \pm 46.98^{\mathrm{AB}}$ & $379.80 \pm 20.23^{\mathrm{C}}$ & $405.45 \pm 36.57$ & 17.858 & 93.905 \\
SE & $0.52 \pm 0.08^{\mathrm{AB}}$ & $0.77 \pm 0.55^{\mathrm{C}}$ & $0.87 \pm 0.66$ & 110.903 & 0.000 \\
WASO, min & $134.12 \pm 44.30^{\mathrm{AB}}$ & $45.05 \pm 24.12$ & $34.70 \pm 17.58$ & 58.614 & 0.000 \\
NREM-I, \% & $15.91 \pm 8.08^{\mathrm{B}}$ & $13.15 \pm 4.93^{\mathrm{C}}$ & $8.40 \pm 2.56$ & 8.783 & 0.000 \\
NREM-2, \% & $66.29 \pm 2.88^{\mathrm{B}}$ & $62.50 \pm 5.45$ & $59.52 \pm 4.41$ & 5.780 & 0.001 \\
NREM-3, \% & $7.60 \pm 4.26^{\mathrm{AB}}$ & $11.25 \pm 4.03^{\mathrm{C}}$ & $14.58 \pm 3.52$ & 14.061 & 0.005 \\
REM, \% & $9.90 \pm 4.62^{\mathrm{AB}}$ & $13.70 \pm 5.51^{\mathrm{C}}$ & $16.96 \pm 3.81$ & 11.014 & 0.000 \\
Arl, times/h & $21.11 \pm 6.32^{\mathrm{AB}}$ & $17.49 \pm 3.69$ & $15.67 \pm 2.58$ & 0.000 \\
AHI, times/h & $2.35 \pm 1.32$ & $2.58 \pm 1.34$ & $2.32 \pm 1.12$ & 0.451 & 0.267 \\
\hline
\end{tabular}

Notes: ${ }^{\mathrm{A}} \mathrm{P}<0.05$ vs IONSD; ${ }^{\mathrm{B}} \mathrm{P}<0.05$ vs healthy controls; ${ }^{\mathrm{C}} \mathrm{P}<0.05$ vs healthy controls.

Abbreviations: PSG, polysomnography; IOSSD, insomnia with objective short sleep duration; IONSD, insomnia with objective normal sleep duration; SOL, sleep onset latency; TST, total sleep time; SE, sleep efficiency; WASO, wake after sleep onset; REM, rapid eye movement sleep; NREM, non REM; Arl, arousal index; AHI, apnea hypopnea index.

that objective short sleep duration, decreased SWS, and increased sleep fragmentation were positively related the activation of NLRP3 inflammasome related proteins.

\section{Discussion}

IOSSD is the most biologically severe phenotype of insomnia and is associated with increased morbidity and mortality. Moreover, patients with IOSSD show poor response to cognitive behavioral therapy. ${ }^{2}$ Maternal anemia in pregnancy is an important risk factor associated with maternal morbidity and perinatal morbidity and mortality. ${ }^{25}$ In a study by Suzanne et al (2018), 50.3\% patients (mean age: $66.1 \pm$ 11.2 years) with insomnia were found to have a sleep duration of $<6 \mathrm{~h} .{ }^{4}$ In the present study, all the participants were young adults (average age: $30.45 \pm 8.12$ years). Among these, $44.4 \%(16 / 36)$ had a sleep duration of $<6 \mathrm{~h}$, as measured by PSG. This indicated that objective short sleep duration is also common in young adults with chronic insomnia.
A

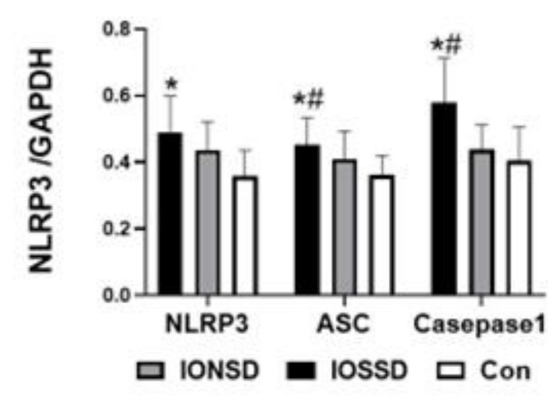

C

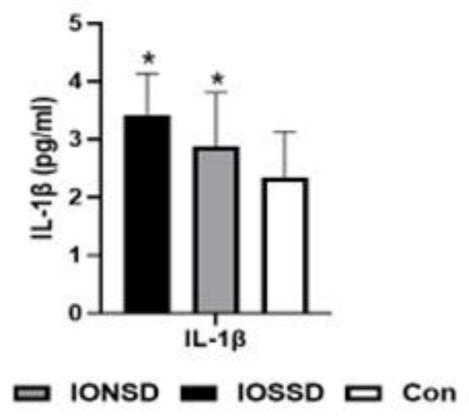

B

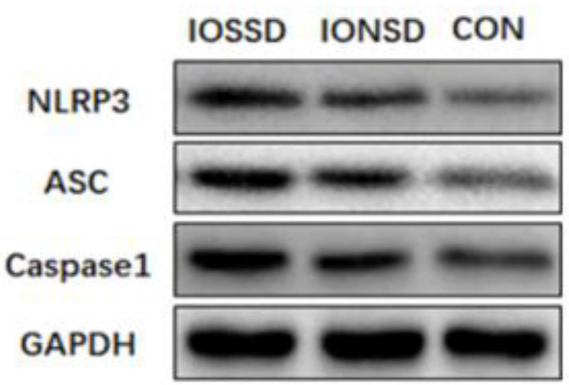

D

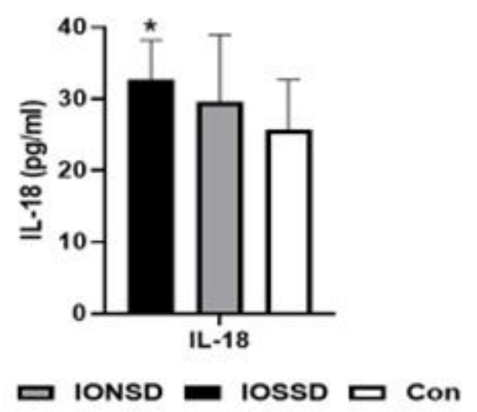

Figure 2 Activation of peripheral NLRP3 inflammasome in insomnia patients with objective short sleep duration. (A and B) Relative expression levels (A) and Western blot bands (B) of peripheral NLRP3, ASC, and caspase I, normalized to the values of GAPDH in IOSSD, IONSD, and healthy controls. (C and D) Serum levels of IL-I $\beta$ (C) and IL18 (D) determined by ELISA. Results are presented as mean \pm standard deviation. One-way ANOVA *P $<0.05$ versus healthy controls; ${ }^{*} \mathrm{P}<0.05$ versus IONSD. Abbreviations: IOSSD, insomnia with objective short sleep duration; IONSD, insomnia with objective normal sleep duration; CON, healthy controls; NLRP3, NOD-like receptor protein 3; ASC, apoptosis-associated speck-like protein; GAPDH, glyceraldehyde phosphate dehydrogenase; IL-I $\beta$, interleukin-I $\beta$; IL-I8, interleukin-I8. 
Table 3 Correlation Matrix Analysis of PSG Parameters and NLRP3 Inflammasome

\begin{tabular}{|l|l|l|l|l|l|}
\hline Variables & NLRP3 & ASC & Caspase-I & IL-I 8 & IL-I $\boldsymbol{\beta}$ \\
\hline SOL & 0.166 & 0.233 & 0.144 & 0.184 & 0.197 \\
TST & $-0.298^{*}$ & $-0.279 *$ & -0.216 & $-0.312^{*}$ & $-0.349 * *$ \\
SE & -0.219 & $-0.320^{*}$ & $-0.310^{*}$ & -0.151 & -0.23 I \\
WASO & $0.389 * *$ & $0.31 I^{*}$ & $0.350^{* *}$ & 0.202 & $0.306^{*}$ \\
NI & $0.345^{* *}$ & 0.251 & -0.058 & $0.333^{*}$ & 0.220 \\
N2 & 0.195 & 0.197 & $0.29 I^{*}$ & 0.254 & 0.083 \\
N3 & $-0.420^{* *}$ & $-0.324^{*}$ & -0.043 & $-0.419^{* *}$ & -0.236 \\
REM & -0.233 & -0.210 & -0.244 & $-0.312^{*}$ & -0.127 \\
Arl & $0.29 I^{*}$ & $0.282^{*}$ & 0.092 & 0.211 & $0.348^{* *}$ \\
\hline
\end{tabular}

Notes: Pearson correlation coefficients $(r)$ are presented; $* \mathrm{P}<0.05, * * \mathrm{P}<0.01$.

In the present study, patients with IOSSD not only had shorter sleep duration, but also showed higher WASO and ArI, lower SWS proportion and SE compared with IONSD and healthy controls. This indicates that nocturnal arousal and related sleep fragmentation are serious problems in IOSSD. Considering the importance of SWS and sleep continuity in the regulation of neuroendocrine and immune systems, ${ }^{26}$ it is entirely plausible that the change in sleep duration and pattern may be partly responsible for the activation of inflammatory reaction in IOSSD.

The second main finding of this study was the increased expressions of NLRP3 related proteins including ASC, caspase-1, and IL-18 in patients with IOSSD. We analyzed the relationship between PSG parameters and NLRP3 related proteins; the results showed that objective short sleep duration, decreased SWS, and increased sleep fragmentation were positively related to the activation of NLRP3 related proteins.

Potential mechanisms linking sleep loss and NLRP3 inflammasome may include the following:

1. Insomnia, especially short sleep duration, is related to the activation of sympathetic system, as evidenced by increased levels of epinephrine and norepinephrine. ${ }^{27-29}$ Changes in the levels of these two neuromediators may be partly responsible for the activation of NLRP3 inflammasome through stimulation of leukocyte adrenergic receptors (eg, ADRB2 and Toll-like-receptor-4) and activation of the nuclear factor (NF)- $\kappa \mathrm{B}$-mediated inflammatory signal pathway. ${ }^{30,31}$

2. SWS plays an important role in adaptive immune memory. ${ }^{26}$ Reduction in SWS may activate the hypothalamus-pituitary-adrenal axis and glucocorticoid receptors in leukocytes, leading to a significant inhibition of antiviral gene programs [eg, interferon regulatory factor (IRF)-mediated type I interferon (IFN) transcription] and the transformation of Th1 to Th2. ${ }^{26}$ Suppression of adaptive immune response may enhance the susceptibility of sleep-deprived patients to infectious diseases, ${ }^{32,33}$ and infectious pathogens are the main activators of NLRP3 inflammasome. ${ }^{31}$

3 . The consumption of oxygen and production of reactive oxygen species (ROS) decrease significantly during sleep; however continual sleep disruption increases the production of $\mathrm{ROS}^{34}$ Considering that ROS is one of the main triggers of NLRP3 inflammasome activation, ${ }^{31}$ it is reasonable to speculate that NLRP3 may be activated through ROS pathway in patients with short sleep duration and poor sleep continuity.

In conclusion, this preliminary study demonstrates the activation of NLRP3 inflammasome in patients with IOSSD. Activation of NLRP3 was positively related to the short sleep duration and sleep fragmentation. Some limitations of this study need to be considered while interpreting the results: 1) The cross-sectional study design does not permit any causal inferences about the relationship between sleep and NLRP3. Prospective studies are required to evaluate the effect of sleep intervention on NLRP3. 2) For ethical reasons, we did not evaluate the expressions of NLRP3 inflammasome in the brain, which can directly reflect the effect of sleep on neuroinflammation. 3) Owing to the small sample size, we could not perform sub-group analysis based on the characteristics of insomnia (difficulty in initiating sleep or maintaining sleep).

\section{Disclosure}

The authors report no conflicts of interest in this work. 


\section{References}

1. Morin CM, LeBlanc M, Daley M, Gregoire JP, Mérette C. Epidemiology of insomnia: prevalence, self-help treatments, consultations, and determinants of help-seeking behaviors. Sleep Med. 2006;7(2):123-130. doi:10.1016/j.sleep.2005.08.008

2. Vgontzas AN, Fernandez-Mendoza J, Liao D, Bixler EO. Insomnia with objective short sleep duration: the most biologically severe phenotype of the disorder. Sleep Med Rev. 2013;17(4):241-254. doi:10.1016/j.smrv.2012.09.005

3. Grandner MA, Patel NP, Gehrman PR, Perlis ML, Pack AI. Problems associated with short sleep: bridging the gap between laboratory and epidemiological studies. Sleep Med Rev. 2010;14(4):239-247. doi:10.1016/j.smrv.2009.08.001

4. Bertisch SM, Pollock BD, Mittleman MA, et al. Insomnia with objective short sleep duration and risk of incident cardiovascular disease and all-cause mortality: sleep heart health study. Sleep 2018;41(6):zsy047. doi:10.1093/sleep/zsy047

5. Vgontzas AN, Liao D, Pejovic S, Calhoun S, Karataraki M, Bixler EO. Insomnia with objective short sleep duration is associated with type 2 diabetes: a population-based study. Diabetes Care. 2009;32(11):1980-1985. doi:10.2337/dc09-0284

6. Jarrin DC, Alvaro PK, Bouchard MA, Jarrin SD, Drake CL, Morin CM. Insomnia and hypertension: a systematic review. Sleep Med Rev. 2018;41:3-38. doi:10.1016/j.smrv.2018.02.003

7. Tobaldini E, Fiorelli EM, Solbiati M, Costantino G, Nobili L, Montano N. Short sleep duration and cardiometabolic risk: from pathophysiology to clinical evidence. Nat Rev Cardiol. 2019;16 (4):213-224. doi:10.1038/s41569-018-0109-6

8. Khassawneh BY, Bathgate CJ, Tsai SC, Edinger JD. Neurocognitive performance in insomnia disorder: the impact of hyperarousal and short sleep duration. J Sleep Res. 2018;27(6):e12747. doi:10.1111/ jsr. 12747

9. Fernandez-Mendoza J, Shea S, Vgontzas AN, Calhoun SL, Liao D, Bixler EO. Insomnia and incident depression: role of objective sleep duration and natural history. J Sleep Res. 2015;24(4):390-398. doi: $10.1111 /$ jsr.12285

10. Zielinski MR, Davis JM, Fadel JR, Youngstedt SD. Influence of chronic moderate sleep restriction and exercise on inflammation and carcinogenesis in mice. Brain Behav Immun. 2012;26(4):672-679. doi:10.1016/j.bbi.2012.03.002

11. Cooper LN, Mishra I, Ashley NT. Short-term sleep loss alters cytokine gene expression in brain and peripheral tissues and increases plasma corticosterone of zebra finch (taeniopygia guttata). Physiol Biochem Zool. 2019;92(1):80-91. doi:10.1086/701170

12. Tang Y, Preuss F, Turek FW, Jakate S, Keshavarzian A. Sleep deprivation worsens inflammation and delays recovery in a mouse model of colitis. Sleep Med. 2009;10(6):597-603. doi:10.1016/j. sleep.2008.12.009

13. Fernandez-Mendoza J, Baker JH, Vgontzas AN, Gaines J, Liao D, Bixler EO. Insomnia symptoms with objective short sleep duration are associated with systemic inflammation in adolescents. Brain Behav Immun. 2017;61:110-116. doi:10.1016/j.bbi.2016.12.026

14. Irwin MR, Wang M, Campomayor CO, Collado-Hidalgo A, Cole S. Sleep deprivation and activation of morning levels of cellular and genomic markers of inflammation. Arch Intern Med. 2006;166 (16):1756-1762. doi:10.1001/archinte.166.16.1756

15. Irwin MR, Witarama T, Caudill M, Olmstead R, Breen EC. Sleep loss activates cellular inflammation and signal transducer and activator of transcription (STAT) family proteins in humans. Brain Behav Immun. 2015;47:86-92. doi:10.1016/j.bbi.2014.09.017

16. Swanson KV, Deng M, Ting JP. The NLRP3 inflammasome: molecular activation and regulation to therapeutics. Nat Rev Immunol. 2019;19(8):477-489. doi:10.1038/s41577-019-0165-0
17. Rovira-Llopis S, Apostolova N, Bañuls C, Muntané J, Rocha M, Victor VM. Mitochondria, the NLRP3 inflammasome, and sirtuins in type 2 diabetes: new therapeutic targets. Antioxid Redox Signal. 2018;29(8):749-791. doi:10.1089/ars.2017.7313

18. Song L, Pei L, Yao S, Wu Y, Shang Y. NLRP3 inflammasome in neurological diseases, from functions to therapies. Front Cell Neurosci. 2017;11:63. doi:10.3389/fncel.2017.00063

19. Hoseini Z, Sepahvand F, Rashidi B, Sahebkar A, Masoudifar A, Mirzaei H. NLRP3 inflammasome: its regulation and involvement in atherosclerosis. J Cell Physiol. 2018;233(3):2116-2132. doi:10.1002/ jcp. 25930

20. Zielinski MR, Gerashchenko D, Karpova SA, et al. The NLRP3 inflammasome modulates sleep and NREM sleep delta power induced by spontaneous wakefulness, sleep deprivation and lipopolysaccharide. Brain Behav Immun. 2017;62:137-150. doi:10.1016/j.bbi.2017.01.012

21. Yu C, Liu Y, Sun L, et al. Chronic obstructive sleep apnea promotes aortic remodeling in canines through $\mathrm{miR}-145 / \mathrm{Smad} 3$ signaling pathway. Oncotarget. 2017;8(23):37705-37716. doi:10.18632/ oncotarget.17144

22. Xia M, Li X, Yang L, et al. The ameliorative effect of fluoxetine on neuroinflammation induced by sleep deprivation. $J$ Neurochem. 2018;146(1):63-75. doi:10.1111/jnc. 14272

23. American Psychiatric Association. Diagnostic and Statistical Manual of Mental Disorders. 5th ed. Washington: American Psychiatric Association; 2013:362-368.

24. Berry RB, Budhiraja R, Gottlieb DJ, et al. Rules for scoring respiratory events in sleep: update of the 2007 AASM manual for the scoring of sleep and associated events. Deliberations of the sleep apnea definitions task force of the American academy of sleep medicine. J Clin Sleep Med. 2012;8(5):597-619. doi:10.5664/jcsm.2172

25. Smith C, Teng F, Branch E, Chu S, Joseph KS. Maternal and perinatal morbidity and mortality associated with anemia in pregnancy. Obstet Gynecol. 2019;134(6):1234-1244. doi:10.1097/ aog.0000000000003557

26. Irwin MR. Why sleep is important for health: a psychoneuroimmunology perspective. Ann Rev Psychol. 2015;66 (1):143-172. doi:10.1146/annurev-psych-010213-115205

27. Vgontzas AN, Tsigos C, Bixler EO, et al. Chronic insomnia and activity of the stress system: a preliminary study. $J$ Psychosom Res. 1998;45(1):21-31. doi:10.1016/s0022-3999(97)00302-4

28. Floam S, Simpson N, Nemeth E, Scott-Sutherland J, Gautam S, Haack M. Sleep characteristics as predictor variables of stress systems markers in insomnia disorder. J Sleep Res. 2015;24(3):296-304. doi: $10.1111 /$ jsr. 12259

29. D'Aurea C, Poyares D, Piovezan RD, Passos G, Tufik S, Mello MT. Objective short sleep duration is associated with the activity of the hypothalamic-pituitary-adrenal axis in insomnia. Arq Neuropsiquiatr. 2015;73(6):516-519. doi:10.1590/0004$282 \times 20150053$

30. Irwin MR, Wang M, Ribeiro D, et al. Sleep loss activates cellular inflammatory signaling. Biol Psychiatry. 2008;64(6):538-540. doi:10.1016/j.biopsych.2008.05.004

31. Kelley N, Jeltema D, Duan Y, The HY. NLRP3 inflammasome: an overview of mechanisms of activation and regulation. Int J Mol Sci. 2019;20(13):3328. doi:10.3390/ijms20133328

32. Patel SR, Malhotra A, Gao X, Hu FB, Neuman MI, Fawzi WW. A prospective study of sleep duration and pneumonia risk in women. Sleep. 2012;35(1):97-101. doi:10.5665/sleep.1594

33. Prather AA, Janicki-Deverts D, Hall MH, Cohen S. Behaviorally assessed sleep and susceptibility to the common cold. Sleep. 2015;38(9):1353-1359. doi:10.5665/sleep.4968

34. Noguti J, Andersen ML, Cirelli C, Ribeiro DA. Oxidative stress, cancer, and sleep deprivation: is there a logical link in this association? Sleep Breath. 2013;17(3):905-910. doi:10.1007/ s11325-012-0797-9 


\section{Publish your work in this journal}

Nature and Science of Sleep is an international, peer-reviewed, open access journal covering all aspects of sleep science and sleep medicine, including the neurophysiology and functions of sleep, the genetics of sleep, sleep and society, biological rhythms, dreaming, sleep disorders and therapy, and strategies to optimize healthy sleep.
The manuscript management system is completely online and includes a very quick and fair peer-review system, which is all easy to use. Visit http://www.dovepress.com/testimonials.php to read real quotes from published authors. 